\title{
Humanização em saúde na graduação em medicina e sua correlação com atividades da IFMSA Brazil
}

\author{
Humanization in healthcare at a medical undergraduate course and its \\ correlation with IFMSA Brazil activities
}
Humanización en salud en la graduación de medicina y su correlación con las actividades de IFMSA Brazil

\begin{abstract}
Valéria Carolina Armas Villegas ${ }^{1}$, Andressa Luciani Pereira Rodrigues ${ }^{2}$
${ }^{1}$ Acadêmica de Medicina da Faculdades Pequeno Príncipe. Curitiba, Paraná

${ }^{2}$ Acadêmica de Medicina da Faculdades Pequeno Príncipe. Curitiba, Paraná
\end{abstract}

\section{RESUMO}

Ao correlacionar humanização e graduação de medicina, estudantes filiados à Federação Internacional das Associações dos Estudantes de Medicina do Brasil (IFMSA Brazil), realizam atividades com o intuito de disseminar e implementar conhecimentos adquiridos em sua formação. O artigo objetiva identificar como a temática 'Humanização em Saúde’ é desenvolvida nos programas da Federação, correlacionando-os com literatura científica. Realizou-se uma revisão integrativa e, posteriormente, analisou-se os relatórios referentes aos programas. Os artigos abordavam formas de inserir a humanização na graduação de medicina. Em relação aos programas, 15 possuíam as palavras 'Humanização', 'Arte' e/ou 'Empatia'. Reforça-se a importância da graduação médica com olhar holístico, voltado para o método clínico centrado na pessoa. A abordagem literária foi aplicada tanto pelo professor na graduação, em literatura

Autor de Correspondência:

*Valéria Carolina Armas Villegas. E-mail: valeria.armas.villegas@hotmail.com 
científica, como por alunos da Federação. Evidencia-se que a humanização em saúde é abordada tanto em atividades curriculares como em extracurriculares na graduação de medicina.

Palavras-chave: Humanização da Assistência. Educação de Graduação em Medicina. Educação Médica.

\begin{abstract}
When correlating humanization and the medical undergraduate course, students affiliated to the International Federation of Medical Students' Associations- Brazil (IFMSA Brazil) carry out activities to disseminate and implement the knowledge acquired in their undergraduate course. The objective of the study was to identify how 'Humanization in Healthcare' is developed in IFMSA Brazil's programs, correlating this with the scientific literature. First, an integrative review was performed, and then the reports related to the programs were analyzed. The articles addressed ways of inserting humanization in medical undergraduate courses. Regarding the programs, 15 had the words 'Humanization', 'Art' and/or 'Empathy'. The importance of the medical undergraduate course with a holistic view, focused on the person-centered clinical method, is reinforced. The literary approach was applied both by the professor at undergraduate level, in scientific literature, and by students of the Federation. It is evident that humanization in healthcare is addressed both in curricular and in extracurricular activities in medical school.
\end{abstract}

Keywords: Humanization of Assistance. Education, Medical, Undergraduate. Education, Medical.

\title{
RESUMEN
}

Al correlacionar humanización y graduación médica, los estudiantes afiliados a la Federación Internacional de las Asociaciones de los Estudiantes de Medicina de Brasil (IFMSA Brazil), realizan actividades con el intento de difundir e implementar los conocimientos adquiridos en su formación. Este trabajo objetiva identificar cómo se desarrolla la 'Humanización en salud' en los programas de IFMSA Brazil correlacionándolos con la literatura. Se realizó una búsqueda en la base de datos BVS y, posteriormente, se analizaron los informes de los programas. Los artículos abordaron formas de insertar la humanización en la graduación. En cuanto a los programas, 15 contenían las palabras "Humanización”, “Arte” y/o “Empatía”. Se refuerza la importancia de la enseñanza médica con enfoque en el método clínico que prioriza la persona. Es evidente que la humanización en salud se aborda en actividades curriculares y extracurriculares en la escuela médica.

Palabras clave: Humanización de la Atención. Educación de Pregrado en Medicina. Educación Médica. 


\section{INTRODUÇÃO}

A temática da humanização em saúde foi reforçada no Sistema Único de Saúde (SUS) em 2003 por meio da Política Nacional de Humanização $(\mathrm{PNH})^{1}$. O objetivo essa política é melhorar a efetividade dos princípios do SUS (universalização, equidade e integralidade) no cotidiano da Atenção Primária no Brasil $^{1}$. Nesse contexto, a humanização se trata da valorização dos usuários, trabalhadores e gestores que compõem o sistema de saúde ${ }^{1}$. São citados como valores dessa política a autonomia e o protagonismo dos sujeitos, a corresponsabilidade mútua, a criação de vínculos solidários, entre outros ${ }^{1}$.

No contexto do cuidado, muito se discute acerca da humanização dentro da área médica, e uma das metodologias para tal é a medicina centrada no paciente, denominada por alguns autores como novo método clínico ${ }^{2}$. Nesse sentido, pode-se resumir os principais componentes da medicina centrada no paciente: cuidado da pessoa; identificação de suas ideias e emoções a respeito do adoecer e a resposta a elas; identificação de objetivos comuns entre médicos e pacientes sobre a doença e sua abordagem, com compartilhamento de decisões e responsabilidades ${ }^{2}$.

Além disso, o método clínico centrado no paciente foi associado a melhora do status de saúde (menos desconforto, menos preocupações e melhora da saúde mental) e maior eficiência de cuidado (menos testes diagnósticos) ${ }^{3}$. A educação médica deve ir além de habilidades teóricas e encorajar os profissionais a considerarem a experiência do paciente integralmente ${ }^{3}$. Dessa forma, os serviços de saúde devem reconhecer que a eficiência advém da prática centrada no paciente e encorajar essa prática por meio de estruturas que implementem a continuidade da relação médico-paciente ${ }^{3}$.

A educação médica no Brasil sofreu forte impacto do Relatório Flexner, publicado em 1910 nos Estados Unidos ${ }^{4}$. Por meio deste relatório o ensino médico se tornou mais pautado no mecanicismo, individualismo, especialização, tecnificação do cuidado à saúde e exclusão de práticas alternativas e curativas, compondo a abordagem biomédica que se tornou futuro contraponto ao cuidado centrado na pessoa, abordado anteriormente ${ }^{4}$.

A abordagem mais recente que dita os moldes da educação médica são as novas Diretrizes Curriculares Nacionais do Curso de Medicina que abordam a importância de formação profissional integral, pautada no humanismo e responsabilidade social $^{5}$. Essas diretrizes conversam com as competências listadas pelo Royal College os Physicians and Surgeons do Canadá, as chamadas CanMEDs, as quais destacam a importância de um profissional comunicador, colaborador, líder, que advoga pela saúde, sabe trabalhar em equipe e exerce a medicina baseada em evidências $(\mathrm{MBE})^{6}$. Reforça-se, portanto, a importância de graduação médica que forme estudante com habilidades que serão aplicadas em seu cotidiano e não apenas teóricas, desligadas da prática clínica.

Ao realizar uma ponte entre a Humanização e a graduação de medicina, estudantes de medicina filiados à Federação Internacional das Associações dos Estudantes de Medicina do Brasil (IFMSA Brazil), por meio de eixos temáticos (Educação Médica; Saúde Pública; Ética e Direitos Humanos em Saúde; e Saúde e Direitos Sexuais e Reprodutivos), realizam atividades com o objetivo de disseminar e implementar conhecimentos adquiridos em sua graduação ${ }^{7}$. A Ficha de Submissão de Atividades (FISA) é submetida em um programa na plataforma SOLAR ${ }^{7}$. Uma FISA é submetida antes da atividade (FISA um) e outra é submetida após sua realização $(\text { FISA dois })^{7}$.

Essas FISAs são corrigidas pelos Coordenadores Nacionais de Programa (CNPs), estudantes de medicina selecionados pelos seus conhecimentos prévios na temática abordada ${ }^{7}$. São ao todo 17 programas, representando as frentes temáticas da Federação ${ }^{7}$. Cada CNP realiza relatório anual das 
atividades corrigidas ${ }^{7}$. Dessa forma, foi realizado mapeamento de atividades ligadas à temática humanização em saúde por meio de relatórios dos Programas "Recursos Humanos e Humanização em Saúde" e "Acesso não Discriminatório à Saúde", referente ao primeiro semestre de 20197. Além disso, foi realizada busca em base de dados para correlacionar a forma como a temática é abordada na literatura científica e nas atividades da IFMSA Brazil ${ }^{7}$

O objetivo deste artigo é identificar como a temática Humanização em Saúde é desenvolvida em atividades realizadas por estudantes de medicina ligados à IFMSA Brazil, correlacionando-as com cenários curriculares e extracurriculares presentes na literatura científica sobre o tema.

\section{MÉTODO}

Esta pesquisa é composta por revisão de literatura integrativa combinada com análise de dados da plataforma SOLAR. A revisão integrativa possui uma base ampla metodológica ${ }^{8}$. Dessa forma, neste tipo de revisão, estudos experimentais e não experimentais são permitidos na análise de dados e a combinação de literatura teórica e empírica se dá de maneira abrangente. Sendo assim, pode-se definir conceitos, revisão de teorias e evidências, análise de problemas metodológicos de um tópico particular ${ }^{8}$. Como resultado, a revisão integrativa permite gerar um panorama consistente e compreensível de conceitos complexos, teorias ou problemas de saúde relevantes para a medicina 8 .

Foi realizada busca em base de dados BVS com os descritores "humanização da assistência" e "educação de graduação em medicina", pesquisados previamente na base brasileira de descritores em saúde (DeCS) e combinados com o conector AND. A pergunta de pesquisa foi "Como a humanização em saúde é abordada na graduação em medicina no
Brasil?". Os artigos encontrados foram tabelados por autor, título, revista, ano e país de publicação e breve resumo. A seleção dos artigos foi feita inicialmente pelo título, depois pelo abstract e leitura de texto completo. Artigos excluídos não respondiam a pergunta de pesquisa, não eram artigos publicados em revistas (como teses de mestrado e doutorado) ou não possuíam o Brasil como país de origem. Não foram utilizados filtros de idioma ou tempo.

Em um segundo momento, foi realizada análise dos relatórios referentes aos programas "Recursos Humanos e Humanização em Saúde" e "Acesso Não Discriminatório à Saúde”. Os relatórios possuíam os campos: nome da atividade, comitê local, regional, temática, tipo de atividade, mensuração de impacto, público-alvo, parcerias, pessoas atingidas, observações. Foram consideradas dentro do tema Humanização em Saúde todas as FISAs um e FISAs dois aprovadas que possuíam as palavras 'Humanização', 'Cuidado', 'Arte' e 'Empatia' em seu título ou temática. Apenas FISAs do primeiro semestre de 2019 aprovadas até a primeira quinzena de agosto foram incluídas nesta pesquisa.

Após a coleta dos artigos e seleção das atividades da IFMSA Brazil, foram comparadas as abordagens realizadas pelos alunos da graduação com aquelas relatadas na literatura científica, de modo a traçar paralelo entre as metodologias de aprendizado, sujeito envolvidos e tipos de atividades propostas.

\section{RESULTADOS}

$\mathrm{Na}$ base de dados BVS, foram encontrados ao todo 25 artigos, dos quais apenas 15 foram selecionados para leitura do texto completo. Desses, dois artigos foram excluídos, posteriormente, por não possuírem texto completo disponível. Dos 13 aprovados, após a leitura completa, apenas sete foram selecionados (figura 1). 
Figura 1 - Fluxograma da coleta de dados na base de dados BVS

\section{Como a humanização em saúde é abordada na graduação em medicina no Brasil?}

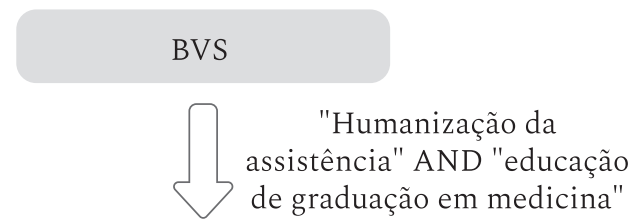

25 artigos

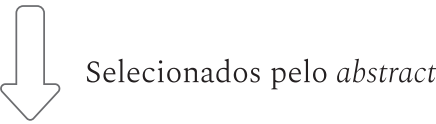

15 artigos

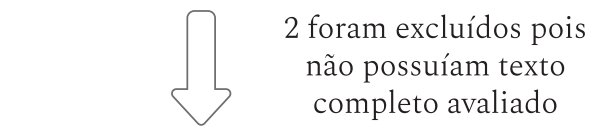

13 artigos

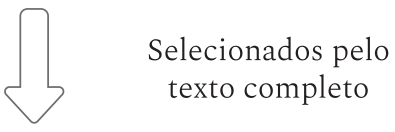

7 artigos
Os artigos selecionados abordavam, principalmente, formas de inserir a humanização na graduação de medicina - 2 traziam propostas extracurriculares (em ação organizada por alunos e em disciplina eletiva) e 5 traziam cenários curriculares. A inserção se deu por meio da correlação de arte e medicina em quatro dos sete artigos selecionados, pela forma de discussão de textos, livros e filmes ${ }^{9-12}$. Também foi abordada, em um artigo, a realização de atividades lúdicorecreativas como forma de aproximar o discente ao paciente e seus familiares ${ }^{10}$. Foram levantadas, na maioria dos artigos selecionados, discussões com os alunos sobre a relação médico-paciente e o processo de adoecimento e morte.

Em relação às FISAs contabilizadas no programa "Recursos Humanos e Humanização em Saúde", das 45 corrigidas até agosto de 2019, 15 possuíam as palavras 'Humanização' (uma FISA), 'Arte' (14 FISAs) e/ou 'Empatia' (uma FISA). Nenhuma FISA continha a palavra 'Cuidado'13. As atividades relacionadas à arte foram as mais encontradas, pois o projeto Sensibilizarte é uma atividade comum neste programa ${ }^{14}$. Esse projeto pode possuir quatro frentes: palhaçoterapia, musicoterapia, artesanato e contação de histórias. Sobre o Programa "Acesso Não Discriminatório à Saúde", foram encontradas três FISAs, das 60 corrigidas até agosto de 2019, sendo duas FISAs relacionadas a cuidado e uma FISA relacionada à empatia (figura 2).

Figura 2 - fluxograma da coleta de dados nos relatórios anuais dos programas

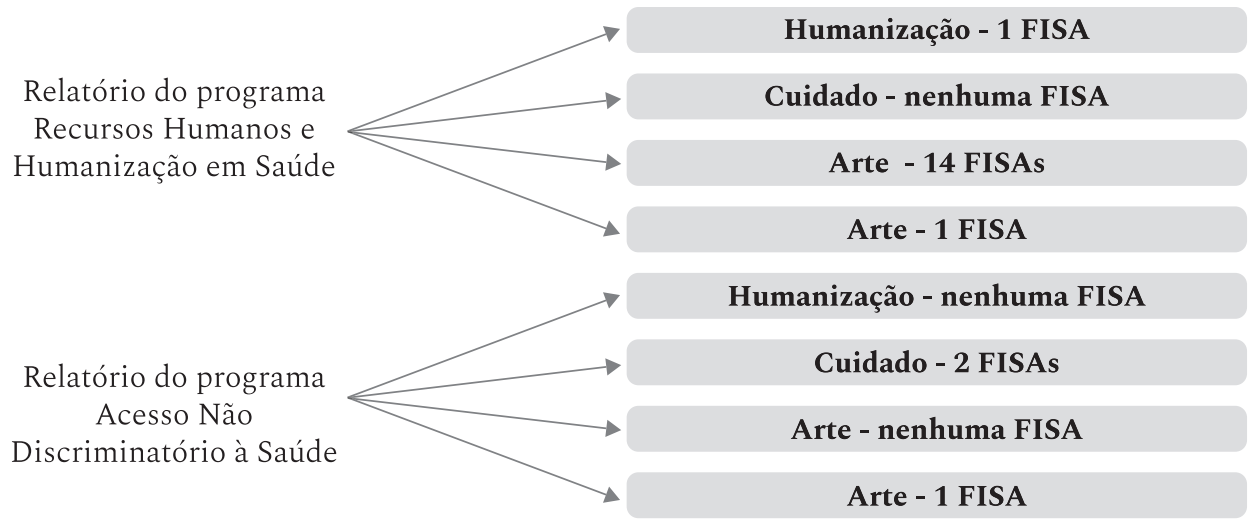

Fonte: autores, 2020. 


\section{DISCUSSÃO}

De acordo com o artigo 3ํ das Diretrizes Curriculares Nacionais do Curso de Graduação em Medicina, o médico formado deve ter um perfil generalista, humanista, crítico e reflexivo ${ }^{5}$. Associada a esse perfil, a capacidade de atuar no processo de saúde e doença, por meio de ações de promoção, prevenção, recuperação e reabilitação em saúde 5 . Dessa forma, o médico deve pautar-se em princípios éticos dentro da perspectiva da integralidade na assistência, do senso de responsabilidade social e do compromisso com a cidadania, atuando como promotor da saúde integral do ser humano ${ }^{5}$.

Nesse sentido, é reforçada a importância de graduação médica com olhar holístico, voltado para o método clínico centrado na pessoa ${ }^{15}$. Constatouse que atividades práticas consolidam a teoria de humanização, tornando-a mais palpável ${ }^{13}$. A forma como os professores médicos se portavam com os pacientes também foi grande exemplo de cuidado e abordagem humanizada para os alunos, reforçando a importância de educadores cientes desta temática ${ }^{13}$.

A correlação arte e medicina é a mais abordada tanto na literatura científica como nas atividades submetidas e aprovadas no programa "Recursos Humanos e Humanização em Saúde”, no primeiro semestre de 2019. Percebe-se que a abordagem literária foi aplicada tanto por professor de graduação na literatura científica como por alunos da IFMSA Brazil em atividade nomeada 'Clube do Livro’ e pela frente de contação de histórias do projeto 'Sensibilizarte'10,14.

Os outros tipos de arte do projeto Sensibilizarte (palhaçoterapia, musicoterapia e artesanato) foram abordados como formas diretas de cuidado, uma vez que o público-alvo são pacientes em hospitais localizados em diversas cidades do Brasil ${ }^{14}$. Logo, troca-se o papel de aluno, para o papel de palhaço, fortalecendo e tornando mais descontraída, desde a graduação, a futura relação médico-paciente.
Segundo Tapajós, R. a finalidade com a qual as Artes são introduzidas nos currículos médicos pode atender à duas perspectivas: a Essencialista que pode ser definida como fonte de experiência estética, de conhecimento e entendimento de si mesmo, dos trabalhos de Arte e do mundo onde eles foram criados, além de desenvolver cultura estética e apreensão de metáforas visuais; e Instrumentalista, a qual valoriza a Arte pela contribuição para o cumprimento de objetivos mais gerais em outras áreas e disciplinas não artísticas. Sobre a perspectiva instrumentalista, educadores médicos podem argumentar que o ensino/aprendizagem das e nas Artes carrega a função de facilitar a execução de outros objetivos mais amplos desse currículo médico, contribuindo para a formação de um profissional detentor de excelência técnica e também de traços humanistas ${ }^{16}$.

Como exemplos dessas perspectivas, Tapajós, R. cita o desenvolvimento e percepção de uma sociedade multicultural, competência em comunicação, desenvolvimento cognitivo, imaginação, julgamento, capacidade de resolução de problemas e pensamentos de complexidade intelectual superior ${ }^{16}$. A educação pelas Artes carrega então, um valor intrínseco e extrínseco utilitário, ambos contribuindo para uma educação generalista e liberal ${ }^{16}$.

A empatia, encontrada em duas FISAs, mostra-se como uma forma de levar a teoria para a prática, uma vez que os acadêmicos de medicina são, não apenas os proponentes das atividades, mas também o seu público-alvo ${ }^{14}$. Por meio de simulações (como de deficiência física e visual, por exemplo) os alunos procuram entender em que situação está o seu paciente, para, posteriormente atendê-lo com mais compreensão ${ }^{14}$. Atividades com essa finalidade foram abordadas no contexto da prática na Atenção Primária, mas não por meio de simulações, nos artigos encontrados ${ }^{17,18}$.

As duas FISAs encontradas sobre cuidado não se correlacionaram com os artigos encontrados 
diretamente, pois não houveram artigos ressaltando recortes populacionais. Os temas das atividades em questão foram cuidado da população negra e cuidado da população de Lésbicas, Gays, Bissexuais, Travestis e Transexuais, Intersexuais (LGBTI+) ${ }^{19}$. A escassez de literatura científica relacionando humanização com minorias e populações vulneráveis, reforça a discriminação como fator de risco para essas populações, já que há a ausência de estudos de suas especificidades. Frente a esta lacuna, reforçadora do acesso à saúde dessas populações, os estudantes de medicina realizam eventos com esses enfoques, com o intuito de complementar a educação médica formal ${ }^{19}$.

\section{CONCLUSÕES}

Visto que o objetivo da pesquisa era correlacionar o conteúdo da literatura sobre humanização em saúde com atividades sobre o tema realizadas na IFMSA Brazil, foi possível constatar que a humanização em saúde é abordada tanto em atividades curriculares como em atividades extracurriculares na graduação de medicina.

Nas atividades curriculares esse aprendizado é adquirido principalmente por meio da atuação docente em campo e atividades teóricas que relacionam a medicina à arte. Nas atividades extracurriculares, o aprendizado dessa temática está mais ligado ao exercício da arte e treinamentos por simulações. Ou seja, um formato complementa o outro.

A correlação entre os artigos publicados e as atividades da IFMSA Brazil submetidas e aprovadas no primeiro semestre de 2019 nos programas "Recursos Humanos e Humanização em Saúde" e "Acesso Não Discriminatório à Saúde" respondeu a pergunta de pesquisa. Se fez possível entendimento do cenário da abordagem de humanização na graduação de medicina, não somente pela visão do docente, mas também do discente.

Percebe-se a importância da inserção da arte no ensino da humanização para acadêmicos de medicina, por meio da reflexão e também da aplicação prática do cuidado e humanização na saúde.

\section{REFERENCIAS}

1. Ministério da Saúde. Cartilha da Política Nacional de Humanização: Humaniza SUS [Internet]. HumanizaSUS. 2010. Available from: http://bvsms.saude.gov.br/bvs/ publicacoes/caderno_textos_cartilhas_politica_ humanizacao.pdf

2. Ribeiro MMF, Amaral CFS. Medicina centrada no paciente e ensino médico: a importância do cuidado com a pessoa e o poder médico. Rev Bras Educ Med. 2008;32(1):90-7.

3. Stewart M, Brown JB, Donner A, McWhinney IR, Oates J, Weston WW, et al. The impact of patient-centered care on outcomes. J Fam Pract. 2000;49(9):796-804.

4. Dias MM de S, Carvalho JL de, Landim LOP, Carneiro C. A Integralidade em Saúde na Educação Médica no Brasil: o Estado da Questão. Rev Bras Educ Med. 2018;42(4):123-33.

5. Ministério da Educação (BR).Resolução CNE/CES nº 04 de 07 novembro de 2001. Dispõe sobre Diretrizes Curriculares Nacionais do Curso de Graduação em Medicina. Diário Oficial da União, Brasília; 2001 p. 1-6

6. Frank JR, Snell L, Sherbino J E. Royal College of Physicians And Surgeons of Canada. CanMEDS 2015 Physician Competency Fram Ottawa R Coll Physicians Surg Canada [Internet]. 2015;1-30. Available from: http:// www.royalcollege.ca/portal/page/portal/rc/canmeds/ resources/publications

7. IFMSA Brazil. Regimento Geral da IFMSA Brazil.

8. Souza MT De, Dias M, Carvalho R De. Revisão integrativa: o que é e como fazer. 2010;8:102-6.

9. Santos MRC, Lins L, Menezes MS. "As intermitências da morte" no ensino da ética e bioética. Rev Bioética. 2018;26(1):135-44. 
10. Amorim KPC, Rocha AKC, Silva ICDS, Melo LMB De, Araújo MAA De. Mediarte com Amor e Humor: uma Experiência a partir do Olhar dos Participantes. Rev Bras Educ Med. 2015;39(2):294-301.

11. Marcello D, Gallian C. Literatura e formação humanística em medicina: o experimento do Laboratório de Humanidades da EPM/UNIFESP. Rev Med [Internet]. 2012;91(3):174-7. Available from: http://www.unifesp. br/centros/cehfi/portal/index.php?option=com content\&view=section\&id=4\&Itemid $=2$

12. Marco MA de, Degiovani MV, Torossian MS, Wechsler R, Joppert SMH, Lucchese AC. Comunicação, humanidades e humanização: A educação técnica, ética, estética e emocional do estudante e do profissional de saúde. Interface Commun Heal Educ. 2013;17(46):683-93.

13. Silva LA, Muhl C, Moliani MM. Ensino Médico E Humanização: Análise a Partir Dos Curriculos De Cursos De Medicina. Psicol Argumento. 2015;33(80):298-309.

14. IFMSA Brasil. Relatório Anual dos Programas. Brasil, 2019

15. Gusso G, Lopes JMC. Tratado de Medicina de Família e Comunidade. Tratado de Medicina de Família e Comunidade. 2012.

16. Tapajós R. A introdução das artes nos currículos médicos. Interface - Comun Saúde, Educ. 2002;6(10):27-36.

17. Rios IC, Sirino CB. A Humanização no Ensino de Graduação em Medicina: o Olhar dos Estudantes. Rev Bras Educ Med. 2015;39(3):401-9.

18. Melo BT, Jordão DA, Beserra KaS, Andrade LSG de, Junior RCC de L, Batista RS de L, et al. Uma Experiência de Integração de Ensino, Serviço e Comunidade de Alunos do Curso de Graduação em Medicina na Atenção Básica no Município de Maceió-AL, Brasil. Rev Ciência Plur. 2017;3(3):69-80.

19. Massignam FM, Bastos JLD, Nedel FB. Discriminação e saúde: um problema de acesso. Epidemiol e Serviços Saúde. 2015;24(3):544-541. 